\title{
Genetic analysis of DNA methylation and gene expression levels in whole blood of healthy human subjects
}

Kristel R van Eijk ${ }^{1,2 \dagger}$, Simone de Jong ${ }^{5 \dagger}$, Marco PM Boks ${ }^{2}$, Terry Langeveld ${ }^{1}$, Fabrice Colas ${ }^{3}$, Jan H Veldink ${ }^{4}$, Carolien GF de Kovel ${ }^{1}$, Esther Janson ${ }^{1}$, Eric Strengman ${ }^{1,5}$, Peter Langfelder ${ }^{6}$, René S Kahn², Leonard $\mathrm{H}$ van den Berg ${ }^{4}$, Steve Horvath ${ }^{6,7}$ and Roel A Ophoff $f^{2,5,6^{*}}$

\begin{abstract}
Background: The predominant model for regulation of gene expression through DNA methylation is an inverse association in which increased methylation results in decreased gene expression levels. However, recent studies suggest that the relationship between genetic variation, DNA methylation and expression is more complex.

Results: Systems genetic approaches for examining relationships between gene expression and methylation array data were used to find both negative and positive associations between these levels. A weighted correlation network analysis revealed that i) both transcriptome and methylome are organized in modules, ii) co-expression modules are generally not preserved in the methylation data and vice-versa, and iii) highly significant correlations exist between co-expression and co-methylation modules, suggesting the existence of factors that affect expression and methylation of different modules (i.e., trans effects at the level of modules). We observed that methylation probes associated with expression in cis were more likely to be located outside CpG islands, whereas specificity for CpG island shores was present when methylation, associated with expression, was under local genetic control. A structural equation model based analysis found strong support in particular for a traditional causal model in which gene expression is regulated by genetic variation via DNA methylation instead of gene expression affecting DNA methylation levels.
\end{abstract}

Conclusions: Our results provide new insights into the complex mechanisms between genetic markers, epigenetic mechanisms and gene expression. We find strong support for the classical model of genetic variants regulating methylation, which in turn regulates gene expression. Moreover we show that, although the methylation and expression modules differ, they are highly correlated.

Keywords: DNA methylation, Gene expression, Association, Epigenetics, WGCNA

\footnotetext{
* Correspondence: ROphoff@mednet.ucla.edu

${ }^{\dagger}$ Equal contributors

${ }^{2}$ Department of Psychiatry, Rudolf Magnus Institute of Neuroscience,

University Medical Center Utrecht, Utrecht 3508, GA, The Netherlands

${ }^{5}$ Center for Neurobehavioral Genetics, University of California Los Angeles,

Box 951761 Gonda \#4357C, 695 Charles E. Young Drive, South Los Angeles,

CA 90095-1761, USA

Full list of author information is available at the end of the article
} 


\section{Background}

Epigenetics has been described as the structural adaptation of chromosomal regions so as to register, signal or perpetuate altered activity states [1]. DNA methylation is one of several forms of epigenetic modifications and involves the covalent binding of a methyl group to a Cytosine-5 at a C-phosphate-G (CpG) site. These sites are relatively rare in the genome but more common at promoter regions of genes, also called $\mathrm{CpG}$ islands (CGIs). CpGs in these islands are less likely to be methylated than $\mathrm{CpGs}$ outside these islands. Recent studies have shown that specifically the CpGs in the shore of CGIs are most frequently involved in differential methylation between tissues or experimental groups [2,3]. Increased methylation of $\mathrm{CpG}$ islands at $5^{\prime}$ end of a gene is associated with gene repression. Possible mechanisms for repression include interference with transcription factor binding or through the recruitment of repressors such as histone deacetylases [4].

Although one would expect DNA methylation at CGIs and expression of the nearby gene to be inversely correlated, this is not necessarily the case. Recent reports also identified positive associations between expression and methylation levels [5-7]. However, negative associations between methylation and expression were found to be enriched particularly in CGIs [6] and promoter regions [5].

Around $30 \%$ of gene expression levels in cell lines [8] and 23\% of DNA methylation levels in blood are heritable [9] and genetic variation associated with expression and methylation levels has been identified in several organisms [6,10-12], tissues [13] and populations [14]. Local (cis) and distal (trans) associations of genetic variation with gene expression levels have been observed. With the arrival of high-throughput DNA methylation assays, methylation quantitative trait loci (mQTLs) can now be studied genome-wide in any tissue or cell type of interest. Similar to expression (eQTLs), more cis than trans regulation has been identified [5-7] but peak enrichment for mQTLs is located in much closer proximity to transcription start sites than that of eQTLs [6].

Attempts to identify three-way associations between genetic variants, expression and methylation on a genome-wide scale in four different brain regions did not identify co-regulation of methylation and expression by the same genetic variants [6], while a study of cerebellar samples did identify three-way associations for a number of genes [7]. In lymphoblastoid cell lines of 77 individuals of the Yoruba Hapmap population, coregulation of expression and methylation levels by the same genetic variants was also found, suggesting a shared mechanism, whereby a genetic variant influences methylation, which in turn influences expression levels [5]. Strong evidence exists that both patterns of CpG methylation $[15,16]$ and gene expression $[13,17,18]$ differ between tissues.

The aims of the current study are i) to relate expression levels to methylation levels, ii) to relate coexpression modules (clusters of expression probes) to co-methylation modules, iii) and to study the relationship between genetic markers, methylation and expression in whole blood of a relatively large $(n=148)$ set of healthy human subjects. For the genetic analysis, we examined the associations of methylation and expression levels and identified genetic markers associated with these levels. To infer directionality in the relationships between genetic variants, methylation and expression, we calculated local edge orienting (LEO) scores based on structural equation models [19]. This method has been applied successfully before and will aid in elucidating the nature of relationship between genetic variation, methylation and expression [20-23].

\section{Results}

Associations between methylation and expression levels

A multivariate linear model analysis for regressing a gene expression level on a methylation level and age and gender resulted in the identification of 522 negative and 276 positive cis associations between methylation and expression levels (False Discovery Rate (FDR) 5\% corrected). A negative association between methylation and transcript level means that increased methylation levels correlate with decreased expression levels, whereas a positive correlation includes levels that both increase or decrease. These associations involved 517 different cis-acting CpG loci (from 461 unique genes) and 495 corresponding expression probes (representing 452 unique genes). For trans effects, we found evidence for 844 negative and 1,806 positive associations between methylation and expression levels involving 705 different methylation probes (from 630 distinct genes), and 170 different expression probes (representing 157 unique genes). Full results are given in Table 1 and Additional file 1: Table S1. Because of the stringent Bonferroni corrections for multiple testing with the number of methylation probes multiplied by the number of expression probes, the effect sizes of surviving trans effects were significantly larger than for cis effects with adjusted explained variance $\left(R^{2}\right)$ ranging from 23 to 60 percent for trans effects and 0.8 to 50 percent for cis regulation (Additional file 2: Figure S1a). Another trend that we observed was that cis effects are enriched for negative correlations $(65.4 \%$ overall) while positive correlations between DNA methylation and gene expression are more frequently observed with trans associations (68.2\%; Fisher's Exact test for count data $p<2.2 \mathrm{e}-16$ ), (Additional file 2: Figure S1b). 
Table 1 Number of probes constituting significant methylation and expression combinations and their association with SNPs

\begin{tabular}{|c|c|c|c|c|c|}
\hline & unique & + & - & overlap & SNP cis \\
\hline & \multicolumn{5}{|c|}{ Cis associations } \\
\hline Methylation & 517 & 224 & 354 & 61 & 69 probes (13.3\%), 86 independent loci \\
\hline \multirow[t]{2}{*}{ Expression } & 495 & 214 & 336 & 55 & 62 probes (12.5\%), 73 independent loci \\
\hline & \multicolumn{5}{|c|}{ Trans associations } \\
\hline Methylation & 705 & 585 & 230 & 110 & 1 probe $(<1 \%)$ \\
\hline Expression & 170 & 101 & 117 & 48 & 0 probes \\
\hline
\end{tabular}

\section{DNA methylation and gene expression are regulated by genetic variants}

Expression levels and methylation levels that were significantly associated with each other were separately tested for regulation by genetic variants. The methylation and expression levels were taken as phenotypes and a linear model of allele dosage, with age and gender as covariates, was tested using PLINK [24]. We focused on local (cis) effects only and observed that approximately $13.7 \%$ of methylation signals and $12.5 \%$ of gene expression levels are associated with single nucleotide polymorphisms (SNPs). Results are given in Table 1, where the number of independent loci, associated with probes, is reported. These were retrieved by pruning the SNPs with an $\mathrm{R}^{2}$ of $>0.2$ to prevent reporting many SNP associations of the same signal due to linkage disequilibrium (LD). Full results are in Additional file 3: Table S2.

\section{Cis-acting methylation sites under genetic control are over-represented in CpG island shores}

We examined the regional distribution of methylation sites $(n=517)$ that are associated with nearby gene expression levels and observed a significant overrepresentation of these loci outside CpG islands and shores compared to all probes present on the Illumina array (50.9\% vs 26\%; Fisher's Exact $p<2.2 \mathrm{e}-16$ ). This coincided with a significant underrepresentation of DNA methylation signal at $\mathrm{CpG}$ islands (13.5\% vs. 42\%, Fisher's Exact $p<2.2 \mathrm{e}-16)$ and a modest increase at the shores flanking CpG islands (35.6\% vs. 32\%, Fisher's Exact $p=0.056)$. The regional distribution of DNA methylation associated with gene expression is somewhat different when DNA methylation is under genetic control. In case of cis genetic regulation we observed a further enrichment of DNA methylation at shores of CpG islands (53.4\%, Fisher's Exact $p=1.3 \mathrm{e}-4)$, whereas trans genetic regulation shows the opposite effect and is less frequently observed for DNA methylation at shores (24.4\%, Fisher's Exact $p=3.9 \mathrm{e}-5)$. The overall results are presented in Table 2 .

\section{Causal relationships between cis-acting methylation and expression probes}

To study the causal relationship between methylation and expression levels that were significantly associated we focused the analysis on pairs of methylation and expression levels with a common cis-acting SNP. We selected the top 20 methylation probes, associated with 19 expression probes that were significantly associated with 147 single common SNPs. Since alleles can be considered fixed features of a genome, we selected SNPs as causal anchors and used a model with residuals of the 20 methylation and 19 expression probes corrected for age and gender. For the causal scenario $\mathrm{SNP} \rightarrow$ Methylation $\rightarrow$ Expression, we found 44 combinations $(29.9 \%)$ with a LEO score above 0.8, involving seven unique genes (Table 3). Of these, 20 combinations have a strikingly high LEO score of 3 or higher; for most of these 20 combinations, the model fitting p-value of the causal model SNP $\rightarrow$ Methylation $\rightarrow$ Expression is above 0.01 , indicating a good fit and lending further credence to these results (Additional file 4: Table S3). For the model SNP $\rightarrow$ Expression $\rightarrow$ Methylation, we found 10 combinations $(6.8 \%)$ with a LEO score above 0.8 , involving again seven unique genes (Table 3 ). The model fitting p-values of these combinations are generally worse (below 0.01), indicating that the linear structural equations models do not fit the data as well and suggesting caution in interpreting the results. A full list of combinations is given in Additional file 4: Table S3. Some SNPs were found to be in high linkage disequilibrium (LD), especially in the Major Histocompatibility Complex (MHC) region on chromosome 6. Therefore only the top SNPs are listed in Table 3. We chosen to investigate these two models since we were interested in the causal direction between DNA methylation and gene expression, after regulation by genetic variation, excluding models 4 and 5 . Model 3, was not informative since we already selected SNPs for association with both methylation and expression.

A locus in the BTN3A2 gene passed the LEO threshold of 0.8 for both models SNP $\rightarrow$ Methylation $\rightarrow$ 
Table 2 Distribution of results over CpG islands and shores

\begin{tabular}{|c|c|c|c|c|c|c|c|c|}
\hline \multirow{2}{*}{$\begin{array}{l}\text { Location } \\
\text { Island }\end{array}$} & \multicolumn{2}{|c|}{$\begin{array}{l}\text { Illumina Human } \\
\text { Methylation } 27\end{array}$} & \multicolumn{2}{|c|}{$\begin{array}{l}\text { Methylation \& } \\
\text { expression cis }\end{array}$} & \multicolumn{2}{|c|}{$\begin{array}{l}\text { Methylation \& } \\
\text { expression trans }\end{array}$} & \multicolumn{2}{|c|}{$\begin{array}{l}\text { Methylation \& } \\
\text { expression \& SNP cis }\end{array}$} \\
\hline & 11,582 & $42 \%$ & 70 & $13.5 \%, p<2.2 \mathrm{e}-16 \downarrow$ & 269 & $38.2 \%, p=0.04$ & 11 & $15.1 \%, p=1.1 \mathrm{e}-06 \downarrow$ \\
\hline Island shore (2kb) & 8,718 & $32 \%$ & 184 & $35.6 \%, p=0.056$ & 172 & $24.4 \%, p=3.9 \mathrm{e}-05 \downarrow$ & 39 & $53.4 \%, p=1.3 \mathrm{e}-04 \uparrow$ \\
\hline Outside island/shore & 7,278 & $26 \%$ & 263 & $50.9 \%, p<2.2 \mathrm{e}-16 \uparrow$ & 264 & $37.4 \%, p=2.5 \mathrm{e}-10 \uparrow$ & 23 & $31.5 \%, \mathrm{~ns}$ \\
\hline Total & 27,578 & & 517 & & 705 & & 73 & \\
\hline
\end{tabular}

Methylation probes were classified into three categories according to UCSC browser (http://genome.ucsc.edu/); CpG islands, CGI shores (up to $2 \mathrm{~kb}$ around an island) and outside islands or shores. Differences compared to Illumina Human Methylation 27K array were tested using Fisher's Exact for count data (Bonferroni threshold $p: 0.05 / 9=0.006$ ). A downward arrow indicates significantly lower percentage of probes while an upward arrow indicates significantly higher percentage of observations compared to the overall probe distribution on the Illumina array.

Expression (LEO score 6.2 based on causal anchor rs9467632) and SNP $\rightarrow$ Expression $\rightarrow$ Methylation (LEO score 1.14 based on causal anchor rs12199613). The two SNPs that were used as causal anchors are in moderate LD $\left(\mathrm{R}^{2}=0.092, \mathrm{D}^{\prime}=0.68\right.$ based on $1000 \mathrm{Gen}$ omes Pilot $1 \mathrm{CEU}$ population [25]. The significant results in both directions could indicate a bi-directional causal interaction between expression and methylation. However, while the model SNP $\rightarrow$ Methylation $\rightarrow$ Expression fits the data well (model fitting p-value $\mathrm{p}=$ 0.10 ), the model SNP $\rightarrow$ Expression $\rightarrow$ Methylation does fits the data poorly (model fitting $\mathrm{p}$-value $\mathrm{p}=6.4 \mathrm{e}-5$ ). Thus, while the evidence for the SNP $\rightarrow$ Methylation $\rightarrow$
Expression model for BTN3A2 is strong, the evidence for the SNP $\rightarrow$ Expression $\rightarrow$ Methylation model is weak.

\section{Weighted correlation network analysis of expression and methylation data}

We separately constructed co-expression and comethylation networks from the expression and methylation data, respectively (Additional file 5: Supplementary Methods), using the Weighted Correlation Network Analysis framework WGCNA [26,27]. In expression data (13,843 genes) we identified 23 co-expression modules (labeled 1-23) with sizes ranging from 32 to 1,520 genes.

Table 3 Top results LEO analysis, Results for top SNPs

\begin{tabular}{|c|c|c|c|c|c|c|c|c|c|}
\hline $\begin{array}{l}\text { Gene } \\
\text { symbol }\end{array}$ & M\&E & CGI & LEO model & $\begin{array}{l}\text { LEO } \\
\text { score }\end{array}$ & P-value & Top SNP & Chr & Bp & Full name \\
\hline BTN3A2 & - & Outside & $S \rightarrow M \rightarrow E$ & 6.90 & 0.15 & rs2093169 & 6 & $26,603,078$ & butyrophilin, subfamily 3 , member A2 \\
\hline$H P$ & - & Outside & $\mathrm{S} \rightarrow \mathrm{M} \rightarrow \mathrm{E}$ & 4.24 & 0.82 & rs8044555 & 16 & $70,710,256$ & haptoglobin \\
\hline CTSW & - & Outside & $\mathrm{S} \rightarrow \mathrm{M} \rightarrow \mathrm{E}$ & 2.73 & 0.13 & rs11227306 & 11 & $65,335,248$ & cathepsin W \\
\hline NAPRT1 & - & Shore & $S \rightarrow M \rightarrow E$ & 2.69 & 0.11 & rs4874159 & 8 & $144,742,093$ & $\begin{array}{l}\text { nicotinate phosphoribosyltransferase } \\
\text { domain containing } 1\end{array}$ \\
\hline PHACS & - & Shore & $\mathrm{S} \rightarrow \mathrm{M} \rightarrow \mathrm{E}$ & 1.50 & $2.9 e-03$ & rs4755227 & 11 & $44,078,659$ & $\begin{array}{l}\text { 1-aminocyclopropane-1-carboxylate } \\
\text { synthase homolog }\end{array}$ \\
\hline PNMA3 & + & Shore & $\mathrm{S} \rightarrow \mathrm{M} \rightarrow \mathrm{E}$ & 1.36 & 0.16 & rs6627737 & $x$ & $151,971,610$ & $\begin{array}{l}\text { nicotinate phosphoribosyltransferase } \\
\text { domain containing } 1\end{array}$ \\
\hline$C D C 16$ & - & Island & $\mathrm{S} \rightarrow \mathrm{M} \rightarrow \mathrm{E}$ & 1.09 & 0.01 & rs11147317 & 13 & $113,957,498$ & $\begin{array}{l}\text { cell division cycle } 16 \text { homolog } \\
\text { (S. cerevisiae) }\end{array}$ \\
\hline HRASLS3 & - & Shore & $S \rightarrow E \rightarrow M$ & 2.42 & 7.2e-04 & rs2030731 & 11 & $63,130,224$ & phospholipase A2, group XVI \\
\hline TACSTD2 & - & Island & $S \rightarrow E \rightarrow M$ & 2.08 & $9.5 \mathrm{e}-03$ & rs11207272 & 1 & $58,846,018$ & $\begin{array}{l}\text { tumor-associated calcium signal } \\
\text { transducer } 2\end{array}$ \\
\hline SRXN1 & - & Shore & $S \rightarrow E \rightarrow M$ & 1.87 & $5.4 \mathrm{e}-03$ & rs6076864 & 20 & 569,825 & sulfiredoxin 1 homolog (S. cerevisiae) \\
\hline C21orf56 & - & Outside & $S \rightarrow E \rightarrow M$ & 1.30 & $2.8 \mathrm{e}-03$ & rs8133866 & 21 & $46,423,604$ & chromosome 21 open reading frame 56 \\
\hline BTN3A2 & - & Outside & $\mathrm{S} \rightarrow \mathrm{E} \rightarrow \mathrm{M}$ & 1.14 & $6.4 \mathrm{e}-05$ & rs12199613 & 6 & $26,475,197$ & butyrophilin, subfamily 3 , member A2 \\
\hline WBSCR27 & - & Shore & $S \rightarrow E \rightarrow M$ & 0.95 & 8.6e-04 & rs11763011 & 7 & $72,922,084$ & $\begin{array}{l}\text { Williams Beuren syndrome chromosome } \\
\text { region } 27\end{array}$ \\
\hline GSTM3 & - & Island & $S \rightarrow E \rightarrow M$ & 0.88 & $1.4 \mathrm{e}-03$ & rs11807 & 1 & $110,062,265$ & glutathione S-transferase mu 3 (brain) \\
\hline
\end{tabular}

This table contains top probes resulting from causality analysis (LEO scores $>0.8$ ). The top seven genes fit the causal scenario SNP $\rightarrow$ Methylation $\rightarrow$ Expression $(\mathrm{S} \rightarrow \mathrm{M} \rightarrow \mathrm{E})$, while the bottom seven genes fit the reverse model in which DNA methylation is regulated by gene expression that is under genetic control

$(\mathrm{S} \rightarrow \mathrm{E} \rightarrow \mathrm{M})$. The Gene Symbol is given in the first column. The second column indicates whether the methylation and expression levels are associated negatively $(-)$ or positively $(+)$. The third column indicates whether the methylation probe is located in a CpG island (CGI), in the shore, or outside both. The columns "LEO model", "LEO score" and "P-value" indicate which causal model fits best with the corresponding LEO score and P-value. This model fitting p-value is calculated using the model chi-square statistic statistic. The chi-square statistic tests the null hypothesis that the model is correct, thus a p-value $>0.01$ indicates good fit. The next column indicates the SNP most significantly associated. The last three columns contain chromosome number and base pair location (NCBI build 36) of the SNP and full name of the gene. 
Additional file 6; Table 1 provides a brief overview of the expression modules along with 10 top hub genes (genes with highest module membership) in each module. A total of 7,743 (56\% of total) genes were assigned to a module while 6,091 background genes were not assigned to a module. Background genes are labeled 0 and colour-coded in grey. Gene ontology (GO) enrichment analysis revealed significant enrichment of multiple modules in various GO terms (Table 4) which provides evidence that these modules are biologically meaningful. A table listing module membership of all genes in expression modules is provided in Additional file 7.
In methylation data $(13,569$ genes $)$ we identified 9 modules of sizes ranging from 37 to 1,067 genes. Additional file 6; Table 2 provides a brief overview of the methylation modules along with 10 top hub genes (genes with highest module membership) in each module. For reader-friendliness, methylation module labels were chosen such that modules with significant overlap with expression modules carry the same label (Methods). A total of 4,088 (30\% of total) genes were assigned to a module, while 9,481 were not assigned. We observed that strong co-expression relationships tend to be more frequent than strong co-methylation. GO enrichment

\section{Table 4 Top GO enrichment terms for expression modules}

\begin{tabular}{|c|c|c|c|c|c|c|}
\hline Module & Size & Rank & p.Bonf & Fraction & Ontology & Term name \\
\hline 1 & 1520 & 1 & $1.20 \mathrm{E}-14$ & 0.65147 & $\mathrm{CC}$ & membrane-bounded organelle \\
\hline 1 & 1520 & 2 & $1.90 \mathrm{E}-14$ & 0.65007 & CC & intracellular membrane-bounded organelle \\
\hline 2 & 703 & 1 & 1.30E-05 & 0.04885 & CC & ribosome \\
\hline 2 & 703 & 2 & $1.50 \mathrm{E}-05$ & 0.07481 & $\mathrm{BP}$ & translation \\
\hline 3 & 658 & 1 & 0.00018 & 0.16382 & CC & extracellular region \\
\hline 4 & 647 & 1 & 0.011 & 0.63711 & CC & membrane-bounded organelle \\
\hline 6 & 442 & 1 & 7.60E-07 & 0.27229 & $\mathrm{BP}$ & response to stress \\
\hline 6 & 442 & 2 & $2.80 \mathrm{E}-06$ & 0.34217 & $\mathrm{BP}$ & signal transduction \\
\hline 7 & 426 & 1 & $9.40 \mathrm{E}-07$ & 0.18734 & $\mathrm{BP}$ & immune system process \\
\hline 8 & 407 & 1 & 0.0042 & 0.66755 & CC & intracellular membrane-bounded organelle \\
\hline 9 & 387 & 1 & $1.70 \mathrm{E}-05$ & 0.82961 & $\mathrm{CC}$ & intracellular part \\
\hline 10 & 355 & 1 & 1.80E-05 & 0.07855 & $\mathrm{BP}$ & ncRNA metabolic process \\
\hline 10 & 355 & 2 & $3.60 \mathrm{E}-05$ & 0.19335 & CC & mitochondrion \\
\hline 12 & 306 & 1 & $2.00 \mathrm{E}-16$ & 0.1134 & CC & ribosome \\
\hline 12 & 306 & 2 & $4.00 \mathrm{E}-16$ & 0.17182 & CC & ribonucleoprotein complex \\
\hline 12 & 306 & 3 & $1.20 \mathrm{E}-14$ & 0.14777 & $\mathrm{BP}$ & translation \\
\hline 12 & 306 & 4 & $2.30 \mathrm{E}-14$ & 0.09278 & $\mathrm{BP}$ & viral transcription \\
\hline 13 & 260 & 1 & 0.00041 & 0.025 & $\mathrm{CC}$ & hemoglobin complex \\
\hline 13 & 260 & 2 & 0.011 & 0.025 & $\mathrm{BP}$ & heme biosynthetic process \\
\hline 14 & 237 & 1 & 0.014 & 0.61086 & MF & protein binding \\
\hline 15 & 118 & 1 & 0.018 & 0.25688 & $\mathrm{BP}$ & intracellular signal transduction \\
\hline 15 & 118 & 2 & 0.032 & 0.12844 & $\mathrm{BP}$ & small GTPase mediated signal transduction \\
\hline 16 & 108 & 1 & 1.30E-05 & 0.18478 & $\mathrm{BP}$ & translation \\
\hline 16 & 108 & 2 & 0.00017 & 0.1087 & $\mathrm{BP}$ & ribosome biogenesis \\
\hline 17 & 99 & 1 & 2.30E-09 & 0.36957 & CC & mitochondrion \\
\hline 18 & 72 & 1 & $3.30 \mathrm{E}-06$ & 0.1791 & $\mathrm{BP}$ & platelet activation \\
\hline 18 & 72 & 2 & $1.20 \mathrm{E}-05$ & 0.22388 & $\mathrm{BP}$ & blood coagulation \\
\hline 19 & 60 & 1 & $1.40 \mathrm{E}-17$ & 0.31034 & MF & structural constituent of ribosome \\
\hline 19 & 60 & 2 & $2.90 \mathrm{E}-17$ & 0.32759 & CC & ribosome \\
\hline 20 & 52 & 1 & 7.10E-23 & 0.32653 & $\mathrm{BP}$ & type I interferon-mediated signaling pathway \\
\hline 20 & 52 & 2 & 7.10E-23 & 0.32653 & $\mathrm{BP}$ & cellular response to type I interferon \\
\hline 23 & 32 & 1 & 0.053 & 0.17241 & CC & external side of plasma membrane \\
\hline
\end{tabular}

For each module we list the top enriched GO terms. Columns list the module label, module size, rank of the enrichment p-value for that particular module, the Bonferroni-corrected enrichment p-value (the correction is performed with respect to the number of GO terms), fraction of the module genes also in the GO term, GO ontology, and GO term name. Multiple expression modules exhibit significant enrichment. Row shading separates modules for easier reading. The enrichment provides evidence that the modules are biologically meaningful. 
analysis of methylation modules revealed multiple significantly enriched categories (Table 5). A table listing module membership of all genes is provided in Additional file 8 .

\section{Preservation of co-expression modules in methylation data and vice versa}

A natural question is whether the expression and methylation modules are related. At the most basic level one can ask whether the expression and methylation modules can be matched based on significant overlap of the genes in each module. We found that expression and methylation modules in general exhibit relatively few overlapping genes (Additional file 9) although some of the overlaps are statistically significant. The most significant overlap $(p=6 e-12)$ is observed between the largest co-expression module and the largest co-methylation module. While the cross-tabulation based module overlap analysis is a simple and intuitive way of assessing module preservation, it has several limitations. In particular, it cannot be used to make strong statements about the lack of module preservation since alternative module detection methods applied to the test data may lead to different results. A rigorous module preservation analysis is based on the network module preservation statistic Zsummary (Methods) since it is independent of the vagaries of detecting modules in test data [28]. We found that the largest expression module 1 (enriched in intracellular-related terms) exhibits moderate preservation, Zsummary $\approx 5$. Modules 9 (enriched in intracellular-related terms), 12 (ribosome), 16 (translation), 17 (mitochondrion), and 19 (ribosome) show weak evidence of preservation, while all other expression modules show no evidence of preservation in methylation data (Zsummary $\leq 2$, Figure $1 \mathrm{~A})$. For the methylation modules we found that modules 1 (intracellular) and 2 (lymphocyte activation) show weak to moderate evidence for preservation, while all other modules show no evidence of preservation (Zsummary < 2, Figure 1B). It is known that the Zsummary statistic tends to increase with module size, reflecting the intuition that a preservation signal observed among many genes is more significant than a similar preservation signal observed among only a few genes. To measure relative preservation irrespective of module size, the authors of [28] proposed the use of a rank-based statistic medianRank. Additional file 10 shows the medianRank statistics in this study. The modules with high Zsummary have low (i.e., near top) ranks. Hence, the two preservation statistics offer a largely consistent picture of module preservation, even though they measure very different quantities.

The weak preservation of co-expression modules in methylation data and vice-versa shows that in general modules (clusters) of expression probes do not correspond to modules of methylation probes. However, we found strong correlations between co-expression modules and co-methylation modules as described in the following.

\section{Associations of expression and methylation eigengenes}

Although the composition of co-expression modules is different from that of co-methylation modules, we observed strong correlations of expression and methylation module eigengenes (Figure $1 \mathrm{C}$ ). A module eigengene is a mathematically optimal way of summarizing the levels of a module (Methods). For example, eigengenes of methylation modules 2 and 7 (both enriched in

Table 5 Top GO enrichment terms for methylation modules

\begin{tabular}{|c|c|c|c|c|c|c|}
\hline Module & Size & Rank & p.Bonf & Fraction & Ontology & Term name \\
\hline 32 & 1067 & 1 & $4.20 \mathrm{E}-09$ & 0.1875 & $\mathrm{CC}$ & extracellular region \\
\hline 1 & 1045 & 1 & $8.20 \mathrm{E}-16$ & 0.790123 & CC & intracellular \\
\hline 1 & 1045 & 3 & $1.50 \mathrm{E}-11$ & 0.083333 & $\mathrm{CC}$ & ribonucleoprotein complex \\
\hline 30 & 616 & 1 & $2.50 \mathrm{E}-21$ & 0.34687 & $\mathrm{BP}$ & anatomical structure development \\
\hline 30 & 616 & 2 & $1.60 \mathrm{E}-18$ & 0.189509 & $\mathrm{BP}$ & nervous system development \\
\hline 7 & 594 & 1 & $8.30 \mathrm{E}-10$ & 0.157424 & $\mathrm{BP}$ & immune system process \\
\hline 7 & 594 & 2 & $9.10 \mathrm{E}-10$ & 0.177102 & MF & receptor activity \\
\hline 3 & 427 & 1 & $3.20 \mathrm{E}-12$ & 0.259947 & $\mathrm{CC}$ & extracellular region \\
\hline 12 & 130 & 1 & 0.0032 & 0.075 & $\mathrm{BP}$ & DNA recombination \\
\hline 2 & 105 & 1 & $1.70 \mathrm{E}-10$ & 0.191919 & $\mathrm{BP}$ & lymphocyte activation \\
\hline 2 & 105 & 2 & $2.30 \mathrm{E}-10$ & 0.20202 & $\mathrm{BP}$ & leukocyte activation \\
\hline 2 & 105 & 3 & $3.60 \mathrm{E}-10$ & 0.323232 & $\mathrm{BP}$ & immune system process \\
\hline
\end{tabular}

For each module we list the top enriched GO terms. Columns list the module label, module size, rank of the enrichment $\mathrm{p}$-value for that particular module, the Bonferroni-corrected enrichment p-value (the correction is performed with respect to the number of GO terms), fraction of the module genes also in the GO term, $\mathrm{GO}$ ontology, and GO term name. Multiple methylation modules exhibit significant enrichment. Row shading separates modules for easier reading. Again, the enrichment provides evidence that the modules are biologically meaningful. 


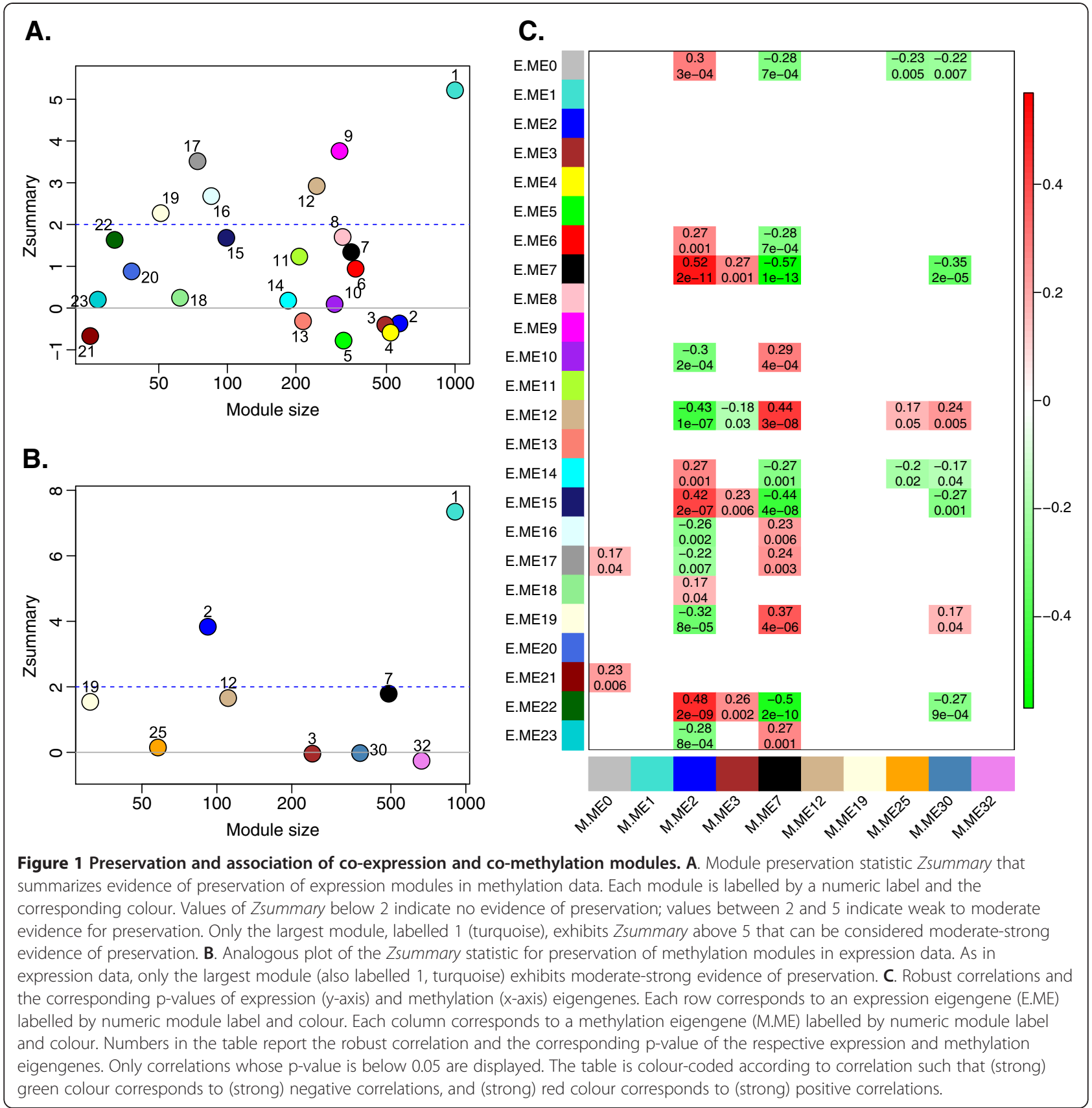

immune system/response terms) are strongly correlated with multiple expression eigengenes such as ME 7 (enriched in immune system process), 12 (ribosome), 15 (intracellular signal transduction), 19 (ribosome), and 22 (no significant enrichment). Methylation module eigengenes 3 (extracellular region) and 30 (anatomical structure morphogenesis, nervous system development) also relate to several expression module eigengenes but the associations are weaker. In summary, we observed multiple strong correlations between expression and methylation module eigengenes.

\section{Module membership of individual genes in expression and methylation modules}

Weighted correlation network methods allow one to define a continuous measure of module membership for each variable in each module as the correlation of the variable profile with the module eigengene (Methods). Additional files 7 and 8 report the module membership (based on expression and methylation profiles) of all genes in all modules. Since the expression and methylation data were measured for the same set of samples, we are able to also provide the module membership of 
expression profiles in methylation modules and vice-versa. These Supplementary Files serve as a resource for relating expression and methylation probes to the modules.

\section{Discussion}

We investigated the relationship between genetic variation, DNA methylation and gene expression in a sample of 148 healthy subjects using array-based data derived from whole blood. We found both negative (levels in opposite direction) and positive (levels in same direction) associations between cis-acting DNA methylation probes and corresponding gene expression levels, confirming previous reports that DNA methylation and gene expression located within a cis-region can be both positively and negatively associated, but are predominantly negative [5-7].

In this study we applied FDR correction for multiple testing for cis associations between methylation and expression, but imposed a more stringent genome-wide significance threshold for trans effects since there is a considerable debate in the literature whether such relationships are reproducible [29,30]. This resulted in a limited number of trans associations that do survive this threshold but with relatively strong effect sizes. It is of note that such trans associations are enriched for positive correlations, whereas traditionally it is expected that methylation and expression are inversely correlated. We hypothesised that these involve genes involved in general methylation pathways, such as genes that induce the attachment of a methyl group. However, a gene ontology analysis did not show any overrepresented pathways (data not shown).

Furthermore, we observed that methylation probes with cis-acting effects on gene expression levels are less likely to be located in $\mathrm{CpG}$ islands and more likely to be present outside CGIs and shores insofar they were not regulated by genetic variation. Tissue- and cell typespecific methylation occurs much more often in gene bodies (outside island and shores) than in CpG island promoters [31], indicating that methylation at $\mathrm{CpG}$ sites in $\mathrm{CpG}$ islands is much more static, which could explain the underrepresentation of $\mathrm{CpG}$ sites associated with expression (and SNPs) in CpG islands. Only for those CpGs that were associated with SNPs, we did concur with previous studies showing more frequent associations with expression in island shores [2,3]. CpG sites located in shores tend to be more variable among individuals and this might lead to an increased number of association findings. In addition, trans associations are less likely to be located in island shores and more likely to be positioned outside CGIs and shores. Also, trans associations are more likely to be positive (67\%).

Identification of genetic variants (SNPs) influencing the methylation and expression levels showed that in more than $12 \%$ of methylation-expression cis-pairs, the methylation and/or the expression level was associated with a SNP in cis, suggesting genetic control of these levels.

Further analysis of genetic regulators (SNPs) of methylation and expression levels investigating the causality revealed three-way causal relationships. Previous studies have attempted to identify three-way associations in various tissues, with mixed results [6,7]. We used local structural equation models to calculate local edge orienting (LEO) scores based on using a cis-acting SNP as causal anchor $[19,32]$. We find that the traditional model of genetic variants regulating methylation, which in turn regulates gene expression to be most common in most of the three-way associations that showed significant evidence for causality (as was hypothesized in literature [5]). The set of genes for which the $S \rightarrow M \rightarrow E$ model fits best does not exhibit significant enrichment for specific functions or pathways. Since the $\mathrm{S} \rightarrow \mathrm{M} \rightarrow \mathrm{E}$ model is expected to be ubiquitous, the lack of enrichment is not surprising. However, one of the genes that fit this model, PNMA3, is located on the $\mathrm{X}$ chromosome. Since inactivation in females may be a confounding factor when analyzing $\mathrm{X}$ chromosomes, we repeated the association analysis for all significant $\mathrm{X}$ chromosomes in males only. We observed no significant differences when using males-only, which confirms that the PNMA3 finding is likely to be true. Strikingly, the reverse model, in which a genetic variant primarily regulates gene expression, which in turn regulates DNA methylation, was the best causal model for a number of genes (including C21ORF56, HRASLS3, TACSTD2, WBSCR27, SRXN1, GSTM3, $B T N 3 A 2)$, although the model p-values of these LEO scores were small, indicating poor fit. For example, one of these genes, C21ORF56, was highlighted in a previous genome-wide study where a three-way association for this gene was identified. Additional experiments indicated that genetic variation in this gene affects chromatin structure in this region [5]. The gene itself may be involved in interindividual differences in response to DNA damaging agents [33]. These mechanisms and our data suggest that loci whereby genetic variation influences expression and in turn methylation may exist and warrants further study. The methylation and expression probes that showed a causal direction in the LEO analysis were all present within the same gene. However, we observed that of all the 798 significant cis associations, only 155 (19\%) involved probes that represent the same gene. This may suggest that the strongest (detectable) causal correlations between DNA methylation and gene expression are likely to be local events.

The systems level analysis afforded by WGCNA reveals that both transcriptome and methylome can usefully be organized into modules. Many co-methylation 
and co-expression modules are highly significantly enriched with gene ontology categories, which provides indirect evidence that these modules are biologically meaningful. Our module preservation analysis between expression and methylation data reveals that most coexpression modules are comprised of genes that do not form a module in the methylation data and vice versa. Only the largest co-expression module shows moderate to strong preservation and overlap with the largest comethylation module. In other words, co-expression modules and co-methylation modules are largely composed of different genes. On the other hand, several pairs of expression and methylation eigengenes show highly significant positive and negative correlations. This suggests the existence of factors that affect expression and methylation of different sets of genes, i.e., trans effects at the module level.

A limiting factor of our study may be the fact that the Illumina $27 \mathrm{k}$ array covers only a selection of CpG sites and is enriched for promoter regions and $\mathrm{CpG}$ islands near genes. Another increasingly important issue is the potential difference between hydroxymethylation and DNA methylation that cannot be distinguished with current methylation arrays $[34,35]$. To date, the role of 5 -hydroxymethylation is not fully understood but it is likely that 5-hydroxymethylation plays a role in demethylation [34-38]. Although there is no reason to assume a systematic influence of 5-hydroxymethylation on our results, we cannot rule this out and further refinement of methylation levels is warranted. A third possible limitation is the use of whole blood comprised of different cell types for our analysis. Yet, although whole blood does not provide the optimal resolution, these cell types can be used to study general genetic mechanisms. Given the sample size we suspect that effects of blood cell composition are limited and do not play a major role in the outcome. We measured gene expression and DNA methylation from the same blood sample so that the composition of different cell types should not substantially affect the overall outcome and conclusions. Moreover, studies have shown that a majority of the strongest eQTLs overlaps between different tissues and cell types $[6,39]$.

\section{Conclusions}

Overall, this study contributes to our understanding about the relationship between genetic markers, methylation and expression levels in whole blood of healthy subjects. We observed cis-associations between methylation and expression levels to be both positive and negative, and most likely to be located outside CGIs and shores. Overrepresentation in shores, as previously found, was only present when selecting methylation/ expression combinations regulated by genetic variation in cis. Methylation/expression combinations in trans are enriched for positive correlations and also located mostly outside CGI's and shores. Results from causality analyses indicate that the conventional model of genetic variants regulating methylation, which in turn regulates gene expression, is most common. This is widely supported in literature [32]. In addition, this indicates that the causal direction analysis is a useful tool for investigating relationships between genotype, methylation and expression. Finally, we showed that methylome and transcriptome are organized into modules. Although the coexpression en co-methylation modules are generally not preserved in one another, we do find highly significant correlations between the modules. These findings suggest that there may be other (trans) factors affecting both methylation and expression, although in different modules. This study encompasses lookup tables for associations between methylation, gene expression, and genotype, as well as methylome and transcriptome modules, for further research.

\section{Methods}

\section{Ethics statement}

All participants gave written informed consent. This study was approved by Medical Research Ethics Committee (MREC) of the University Medical Center Utrecht, The Netherlands.

\section{Pre-processing of genotype, methylation and expression data}

Genotype, methylation and expression data were collected for different numbers of samples. For the 148 healthy subjects eventually analyzed in this paper, data was available for all three layers of genetic information after quality control, as described below. Our final data set consisted of 72 males and 76 females with a mean age of 52 (range: 19-88); all subjects were of Dutch ancestry with at least three of the four grandparents born in The Netherlands.

\section{Genotype SNP data}

Genotype data for subjects was generated on two different array platforms, 105 individuals on Illumina CytoSNP (299,173 SNPs) and 96 on Illumina 300k chips (300,299 SNPs). For each SNP platform, quality control procedures were initially performed separately using PLINK [24]. Subjects were excluded based on $>5 \%$ missing genotypes and gender errors (Additional file 11). We used linkage disequilibrium (LD) based SNP pruning to select the most informative SNPs $\left(\mathrm{R}^{2}<0.2\right)$, only for subsequent quality control steps. This resulted in $\sim 60 \mathrm{k}$ SNPs for both sets to assess heterozygosity $(\mathrm{F}<3$ Standard Deviation (SD)), homozygosity $(\mathrm{F}>3 \mathrm{SD})$ and relatedness by pairwise identity by descent (IBD) values (pihat $>0.1$ ). 
Datasets were merged with Hapmap Phase 3 individuals to check ethnicity (Additional file 12) (ethnic outliers detected by visual inspection). After these QC procedures on subjects (excluding in total 8 individuals) quality control on SNPs was performed as follows. All SNPs were filtered on missingness $(>2 \%)$ and Hardy Weinberg ( $p>1 \mathrm{e}-6)$ before merging the two datasets. 84,367 SNPs were shared between the two datasets. No related samples were detected in the merged datasets (according to criteria described above). We imputed the merged dataset with Hapmap2, release 24 using Beagle [40]. SNPs with an imputation score $>0.8$ and present originally in one or both datasets were extracted and 417,708 SNPs remained for all further analyses.

\section{DNA methylation data}

Methylation data was obtained using Illumina HumanMethylation 27 beadchips for two batches of 105 and 96 healthy subjects. The assay detects methylation status at CpG sites after bisulfate conversion, by means of probes designed for either methylated or unmethylated sequence. Methylation probes were classified into 3 different categories depending on the location of the probe with respect to a CpG island. Based on the UCSC Table browser (http://genome.ucsc.edu/; [41]), NCBIbuild36, categories were defined as $\mathrm{CpG}$ island, $\mathrm{CpG}$ island shore (sequences up to $2 \mathrm{~kb}$ from an island), or outside CpG islands/shores. Ethnical outliers and samples with gender errors in genotype data were removed from the methylation data. Gender was checked by hierarchical clustering of X-chromosomal probes, excluding four individuals. Another three individuals were removed based on detection $p$-values ( $>0.01$ for $>1 \%$ of probes) and 3,027 of 27,578 probes were excluded based on detection values ( $p>0.01$ for $>1 \%$ of the samples). Both channels of the methylation array were quantile normalized independently. Beta values of a probe were calculated by dividing the methylated signal by the sum of the methylated and unmethylated signal. Next, five potential array outliers were removed in an unbiased fashion. Specifically, we used the SampleNetwork $R$ function package [42] to calculate the Interarray based sample connectivity score Z.k. We removed samples with a Z.k value less than -3 since their connectivity is 3 standard deviations below the mean value. Batch effects of dataset, plate, array and position were removed using ComBat [43]. After these procedures, 24,561 probes remained and were mapped to the human genome using the UCSC Human BLAT Search function. In total, 25 probes did not map to the human genome, whereas 338 probes did not map uniquely (mapped more than once), and both these probes have been removed. Moreover, 904 probes that contained a SNP, based on Hapmap release 27, with a minor allele frequency (MAF) > 1\% have been removed as well, leaving a total of 23,294 probes for analyses.

\section{Gene expression data}

Gene expression data was generated in two batches, one on Illumina $\mathrm{H} 8$ beadchip (26 healthy subjects) and one on Illumina H12 beadchip (147 healthy subjects). BeadStudio ${ }^{\circ}$ software version 3.2.3 was used to generate background-corrected gene expression data. Data was normalized, transformed and filtered separately before merging and batch effect removal. Specifically, the datasets were separately quantile normalized and $\log 2$ transformed using the Lumi package for $\mathrm{R}$ [44]. Probes were filtered based on detection values generated by BeadStudio $\odot$. The detection $p$-value threshold was set at 0.01 . This resulted in 17,433 expression probes overlapping between both batches. Batch effects resulting from the use of different arrays at different time points were removed using ComBat [43]. An unbiased analysis based on interarray correlations identified 16 samples from batch 2 as potential outliers, which were subsequently removed from the analysis. Of 17,433 probes, 15,983 mapped to a single genomic location, based on a previous study [45]. In addition, 465 probes contained a SNP, based on Hapmap release 27, with a MAF > 1\% and have been removed, leaving 15,983 probes for analyses.

DNA methylation and gene expression data have been processed using the same blood sample, excluding possible batch effects, such as the effect of different time points.

\section{Identifying cis and trans effects between DNA methylation and gene expression}

We called a methylation probe cis acting with respect to a given gene expression probe if there was a significant association (as defined below) within a 500kb interval between the probes. A methylation probe was called trans acting if it was significantly associated with the expression probe (as defined below) outside the $500 \mathrm{~kb}$ interval.

To determine whether a significant association exists between expression and methylation levels we used a multivariate linear regression model for regressing the gene expression level (dependent variable) on the methylation level (independent variable) with age and gender as covariates. We took methylation levels as independent variable since we are interested in the epigenetic control of gene expression levels. Associations can be positive (DNA methylation levels and gene expression levels both increase or decrease) or negative (increased methylation level corresponds with a decrease in gene expression level and vice versa). The Wald test $p$-value for the association between methylation and expression was used as significance level. Correction of the significance level for 
multiple testing was performed separately for identifying cis acting methylation probes (FDR correction) and trans acting methylation probes (Bonferroni correction).

\section{Identification of cis-and trans-acting SNPs}

Expression levels and methylation levels that were significantly associated with each other were tested for association with SNPs to identify cis-and trans-acting genetic variations. For this analysis, the real and imputed (imputation score $>0.8$ ) genotypes were used, and a MAF threshold of $5 \%$ for these SNPs was set.

Analogous to our previous definition, a SNP significantly associated with a given gene expression or DNA methylation probe was called cis-acting with respect to the probe if the SNP and the probe were within $500 \mathrm{~kb}$ of each other, and trans-acting if they were more than $500 \mathrm{~kb}$ apart.

To determine whether a significant relationship exists between a SNP and a methylation or expression level we again used a multivariate linear regression model for regressing the methylation or expression level (dependent variable) on the SNP (independent variable) with age and gender as covariates. The regressions were performed using the PLINK software [24]. Correction for multiple testing was performed separately for cisacting SNPs (0.05 divided by the number of probes) and trans-acting SNPs (0.05 divided by the number of possible combinations $\left(p<0.05 /\left(\#\right.\right.$ probes $\left.^{*} 417,708\right)$.

\section{Evaluating causal relationships using local edge orienting scores of observed cis effects}

To evaluate the fit of different causal models involving 3 variables (i.e., a cis-acting SNP, a cis-acting methylation probe, and a corresponding expression probe), we calculated the single marker local edge orienting score (LEO. NB.SingleMarker) as described elsewhere [19,32]. In short, a SNP can be used as causal anchor for evaluating the causal relationships between methylation and expression levels if the SNP is associated with at least one of them. We use the SNP as causal anchor for calculating the LEO score since genotypes are fixed at each locus as opposed to variable methylation and expression levels [19]. In this case, one can evaluate the fit of the following five models describing the causal relationships between a SNP (denoted S), a methylation probe (M) and an expression probe (E): model $1: \mathrm{S} \rightarrow \mathrm{M} \rightarrow \mathrm{E}$; model 2: $\mathrm{S} \rightarrow \mathrm{E} \rightarrow \mathrm{M}$; model 3: $\mathrm{M} \leftarrow \mathrm{S} \rightarrow \mathrm{E}$; model $4: \mathrm{S} \rightarrow \mathrm{E} \leftarrow \mathrm{M}$; model 5: $\mathrm{S} \rightarrow \mathrm{M} \leftarrow \mathrm{E}$. For each causal model a chi-square test based model fitting p-value was calculated with the structural equation modelling (SEM) $\mathrm{R}$ package [46]. The relative fit of causal model 1 (SNP $\rightarrow$ Methylation $\rightarrow$ Expression) was assessed using the single anchor local edge orienting score (LEO.SingleMarker), which is the logarithm (base 10) of the ratio of the model fitting $p$-value divided by that of the next best fitting alternative model [19]. Thus a positive LEO.SingleMarker score indicates that the causal model $\mathrm{S} \rightarrow \mathrm{M} \rightarrow \mathrm{E}$ fits the data better than all other competing models. As significance threshold we used the LEO threshold of 0.8 , as recommended in [19] based on extensive simulations as well as empirical studies. We decided to focus on local cis effects since there is considerable debate in the literature whether trans relationships are reproducible [29,30]. Since we were interested in causal direction for predetermined three-way associations, we only selected SNPs associated with both the methylation and expression levels in cis. To protect the causal analysis from biases due to age and gender, we utilized residuals of methylation and expression levels corrected for age and gender in the causal analysis using a linear regression by Limma in $\mathrm{R}$ [47].

\section{Weighted correlation network analysis of gene expression and methylation data}

A detailed description of our correlation module based analyses can be found in Additional file 5. Here we provide a terse summary. Weighted correlation network analysis implemented in the WGCNA R package [26,27] was first applied to the expression data to identify coexpression modules. Co-expresssion modules correspond to clusters of interconnected genes defined as branches of a hierarchical cluster tree. Since modules are defined without respect to gene ontology information they are initially labelled by arbitrary integers and coded by colours. Next WGCNA was applied to the methylation data to find co-methylation modules. For easier interpretation of the relationships between expression and methylation modules, we use the same module labels for modules that show significant overlap. The matching of module labels was performed using the function matchLabels from the WGCNA R package; it is based on significance of module overlaps quantified using Fisher's exact test. Weighted networks have the advantage of preserving the continuous nature of coexpression and co-methylation information, which is particularly useful when studying module preservation. To assess the preservation of expression and methylation modules in the corresponding complementary data set, we use the network module preservation statistics described in [28] and implemented in the function modulePreservation in the WGCNA R package. Network module preservation statistics assess whether the density and connectivity patterns of modules defined in a reference data set are preserved in a test data set. Network preservation statistics do not require that modules be identified in the test data set and hence independent of the ambiguities associated with module identification in the test data set. The permutation test of the 
modulePreservation function leads to a composite module preservation statistic referred to as Zsummary. The Zsummary statistic of a given module summarizes the evidence that the network connections of the module are more significantly preserved than those of random set of genes of equal size. We adopted the following recommended significance thresholds for Zsummary [26-28]: Zsummary $<2$ implies no evidence that the module is preserved, $2<Z$ Zsummary $<10$ implies weak to moderate evidence, and Zsummary $>10$ implies strong evidence for module preservation. Thus, we report $\mathrm{Z}$ summary for each expression and methylation module in the methylation and expression test data sets, respectively.

Since modules group together highly correlated variables, it is advantageous to summarize the variable profiles using a single representative. We use the module eigengene $\mathrm{E}$, defined as the first principal component of the standardized matrix containing the variables in the module. The module eigengene can be intuitively understood as a weighted average of the variable profiles in the module.

\section{Additional files}

Additional file 1: Table S1. Comprises two tables that list all significant methylation and expression associations in cis (S1a), and trans (S1b).

Additional file 2: Figure S1. Are two figures that show the coefficient and explained variance of associations between methylation and expression

Additional file 3: Table S2. Contains tables with all significant cis MQTLs (S2a) and eQTLs (S2b).

Additional file 4: Table S3. Is a table with all LEO results. Combinations that have a LEO score above 0.8 for the model $\mathrm{S}>\mathrm{M}>\mathrm{E}$ are shown in light yellow of which LEO scores above 3 are shown in dark yellow. For the reverse model $(S>E>M)$ combinations with a LEO score above 0.8 are shown in orange. Significant p-values (above 0.01 ) are coloured in green.

Additional file 5: Contains supplementary methods, namely, a more detailed description of Weighted Correlation Network Analysis (WGCNA).

Additional file 6: Is an overview of the modules identified in the expression (Table 1) and methylation (Table 2) data.

Additional file 7: Includes a table of continuous module membership $k M E_{i}$ of all expression profiles in all expression modules. Each row in the table corresponds to one gene expression profile. Columns give the gene Entrez idenitifier, module label, and KME and the corresponding (uncorrected) $\mathrm{p}$-values for each module.

Expression modules are labelled by E.0, E.1, etc.

Additional file 8: Includes a table of continuous module membership $k M E_{i}$ of all methylation profiles in all methylation modules. Each row in the table corresponds to one methylation profile. Columns give the gene Entrez idenitifier, module label, and kME and the corresponding (uncorrected) p-values for each module. Methylation modules are analogously labeled by M.0, M.1, etc.

Additional file 9: Shows the overlap of expression and methylation modules. Each row corresponds to an expression module (labelled by the numeric labels, colours and total number of genes in the module, on the left), and each column corresponds to a methylation module (labelled the numeric labels, colours, and total number of genes in the module, at the bottom). Numbers in the table indicate number of genes in the overlap, and the Fisher exact test $p$-value for the overlap. Only overlaps whose $p$-value is below 0.05 are shown. The table is coloured such that significant overlaps are coloured in strong red colour. Most overlaps are quite small but some are nevertheless statistically highly significant.

Additional file 10: Shows the medianRank statistics for the Module preservation with in (A) preservation of expression modules in methylation data, and in (B) preservation of methylation modules in expression data.

Additional file 11: Is a table with the number of excluded samples per step.

Additional file 12: Is a clusterplot of all samples together with Hapmap phase 3 populations.

\section{Abbreviations}

CpG: Cytosine-Phosphate-Guanine; CGI: CpG Island; QTL: Quantitative Trait Loci; LEO: Local Edge Orienting; FDR: False Discovery Rate; SNP: Single Nucleotide Polymorphism; LD: Linkage Disequilibrium; MHC: Major Histocompatibility Complex; WGCNA: Weighted Gene Co-expression Network Analysis; GO: Gene Ontology; MREC: Medical Research Ethics Committee; SD: Standard Deviation; IBD: Identity By Descent; SEM: Structural Equation Modeling.

\section{Competing interests}

The authors have declared that no competing interests exist.

\section{Authors' contributions}

KRE, SJ and PL wrote the paper. KRE, SJ, TL and PL analyzed the data. MPMB, $\mathrm{SH}$ and RAO designed the study. TL, FC and PL wrote scripts for the analyses. RAO, MPMB, SH, JHV, LHB and RSK provided data, materials, and analysis tools. EJ and ES processed the data. CGFK advised on statistics. The manuscript has been seen and approved by all listed authors.

\section{Acknowledgements}

The authors thank Bobby Koeleman for advice on statistics and Flip Mulder and Yafeng Zhang for bioinformatics support. We thank SARA Computing and Networking Services (www.sara.nl) for their support in using the Lisa Compute Cluster, which facilitates the Genetic Cluster Computer. This study was funded by NIH/NINDS R01 NS058980 and NIH/NIDA DA028526 to R.A.O.

\section{Author details}

'Department of Medical Genetics, University Medical Center Utrecht, Utrecht 3584, CG, The Netherlands. ${ }^{2}$ Department of Psychiatry, Rudolf Magnus Institute of Neuroscience, University Medical Center Utrecht, Utrecht 3508, GA, The Netherlands. ${ }^{3}$ Department of Medical Statistics and Bioinformatics, Leiden University Medical Center, Leiden 2300, RC, The Netherlands. ${ }^{4}$ Department of Neurology, Rudolf Magnus Institute of Neuroscience, University Medical Center Utrecht, Utrecht 3508, GA, The Netherlands. ${ }^{5}$ Center for Neurobehavioral Genetics, University of California Los Angeles, Box 951761 Gonda \#4357C, 695 Charles E. Young Drive, South Los Angeles, CA 90095-1761, USA. ${ }^{6}$ Department of Human Genetics, David Geffen School of Medicine, University of California, Los Angeles, CA 90095, USA.

${ }^{7}$ Department of Biostatistics, School of Public Health, University of California, Los Angeles, CA 90095, USA.

Received: 10 August 2012 Accepted: 30 October 2012 Published: 17 November 2012

\section{References}

1. Bird A: Perceptions of epigenetics. Nature 2007, 447(7143):396-398.

2. Irizarry RA, et al: The human colon cancer methylome shows similar hypo- and hypermethylation at conserved tissue-specific $\mathrm{CpG}$ island shores. Nat Genet 2009, 41(2):178-186.

3. Irizarry RA, Wu H, Feinberg AP: A species-generalized probabilistic modelbased definition of CpG islands. Mamm Genome 2009, 20(9-10):674-680.

4. Sengupta N, Seto E: Regulation of histone deacetylase activities. J Cell Biochem 2004, 93(1):57-67.

5. Bell JT, et al: DNA methylation patterns associate with genetic and gene expression variation in HapMap cell lines. Genome Biol 2011, 12(1):R10. 
6. Gibbs JR, et al: Abundant quantitative trait loci exist for DNA methylation and gene expression in human brain. PLoS Genet 2010, 6(5):e1000952.

7. Zhang $D$, et al: Genetic control of individual differences in gene-specific methylation in human brain. Am J Hum Genet 2010, 86(3):411-419.

8. Monks SA, et al: Genetic inheritance of gene expression in human cell lines. Am J Hum Genet 2004, 75(6):1094-1105.

9. Boks MP, et al: The relationship of DNA methylation with age, gender and genotype in twins and healthy controls. PLoS One 2009, 4(8):e6767.

10. Cookson W, et al: Mapping complex disease traits with global gene expression. Nat Rev Genet 2009, 10(3):184-194.

11. Stranger $B E$, Dermitzakis ET: The genetics of regulatory variation in the human genome. Hum Genomics 2005, 2(2):126-131.

12. Stranger $B E$, et al: Relative impact of nucleotide and copy number variation on gene expression phenotypes. Science 2007, 315(5813):848-853.

13. Nica $A C$, et al: The architecture of gene regulatory variation across multiple human tissues: the MuTHER study. PLOS Genet 2011, 7(2):e1002003.

14. Stranger $B E$, et al: Population genomics of human gene expression. Nat Genet 2007, 39(10):1217-1224.

15. Ghosh S, et al: Tissue specific DNA methylation of CpG islands in normal human adult somatic tissues distinguishes neural from non-neural tissues. Epigenetics: official journal of the DNA Methylation Society 2010 5(6):527-538.

16. Liang $P$, et al: Genome-wide survey reveals dynamic widespread tissuespecific changes in DNA methylation during development. BMC Genomics 2011, 12(1):231.

17. Huang GJ, et al: High resolution mapping of expression QTLs in heterogeneous stock mice in multiple tissues. Genome Res 2009, 19(6):1133-1140.

18. Petretto $E$, et al: Heritability and tissue specificity of expression quantitative trait loci. PLoS Genet 2006, 2(10):e172

19. Aten JE, et al: Using genetic markers to orient the edges in quantitative trait networks: the NEO software. BMC Syst Biol 2008, 2:34

20. Chen $Y$, et al: Variations in DNA elucidate molecular networks that cause disease. Nature 2008, 452(7186):429-435.

21. Inouye $M$, et al: An immune response network associated with blood lipid levels. PLoS Genet 2010, 6(9):e1001113.

22. Plaisier $\mathrm{CL}$, et al: $\mathrm{A}$ systems genetics approach implicates USF1, FADS3, and other causal candidate genes for familial combined hyperlipidemia. PLoS Genet 2009, 5(9):e1000642.

23. Presson AP, et al: Integrated weighted gene co-expression network analysis with an application to chronic fatigue syndrome. BMC Syst Biol 2008, 2:95.

24. Purcell S, et al: PLINK: a tool set for whole-genome association and population-based linkage analyses. Am J Hum Genet 2007, 81(3):559-575.

25. Johnson AD, et al: SNAP: a web-based tool for identification and annotation of proxy SNPs using HapMap. Bioinformatics 2008, 24(24):2938-2939.

26. Langfelder $\mathrm{P}$, Horvath S: WGCNA: an R package for weighted correlation network analysis. BMC Bioinformatics 2008, 9:559.

27. Zhang B, Horvath S: A general framework for weighted gene co-expression network analysis. Stat App/ Genet Mol Biol 2005, 4:Article17.

28. Langfelder $P$, et al: Is my network module preserved and reproducible? PLoS Comput Biol 2011, 7(1):e1001057.

29. Peirce $J$, et al: How replicable are mRNA expression QTL? Mamm Genome 2006, 17(6):643-656

30. van Nas A, et al: Expression quantitative trait loci: replication, tissue- and sex-specificity in mice. Genetics 2010, 185(3):1059-1068.

31. Maunakea AK, et al: Conserved role of intragenic DNA methylation in regulating alternative promoters. Nature 2010, 466(7303):253-257.

32. Horvath S: Structural Equation Models and Directed Networks. In Weighted Network Analysis: Applications in Genomics and Systems Biology. Edited by Horvath S. New York: Springer Book; 2011:279-320.

33. Fry RC, et al: Genomic predictors of interindividual differences in response to DNA damaging agents. Genes Dev 2008, 22(19):2621-2626.

34. Jin SG, Kadam S, Pfeifer GP: Examination of the specificity of DNA methylation profiling techniques towards 5-methylcytosine and 5-hydroxymethylcytosine. Nucleic Acids Res 2010, 38(11):e125.

35. Dahl C, Gronbaek K, Guldberg P: Advances in DNA methylation: 5-hydroxymethylcytosine revisited. Clinica chimica acta; international journal of clinical chemistry 2011, 412(11-12):831-836.
36. Guo JU, et al: Emerging roles of TET proteins and 5-hydroxymethylcytosines in active DNA demethylation and beyond. Cell Cycle 2011, 10(16):2662-2668.

37. Tan L, Shi YG: Tet family proteins and 5-hydroxymethylcytosine in development and disease. Development 2012, 139(11):1895-1902.

38. Branco MR, Ficz G, Reik W: Uncovering the role of 5-hydroxymethylcytosine in the epigenome. Nat Rev Genet 2012 13(1):7-13.

39. Ding J, et al: Gene expression in skin and lymphoblastoid cells: Refined statistical method reveals extensive overlap in cis-eQTL signals. Am J Hum Genet 2010, 87(6):779-789.

40. Browning BL, Browning SR: A unified approach to genotype imputation and haplotype-phase inference for large data sets of trios and unrelated individuals. Am J Hum Genet 2009, 84(2):210-223.

41. Karolchik D, et al: The UCSC Table Browser data retrieval tool. Nucleic Acids Res 2004, 32(Database issue):D493-D496.

42. Oldham MC, Langfelder $P$, Horvath S: Sample Networks for Enhancing Cluster Analysis of Genomic Data: Application to Huntington's Disease. BMC Syst Biol 2011, 6:63.

43. Johnson WE, Li C, Rabinovic A: Adjusting batch effects in microarray expression data using empirical Bayes methods. Biostatistics 2007, 8(1):118-127.

44. Du P, Kibbe WA, Lin SM: lumi: a pipeline for processing Illumina microarray. Bioinformatics 2008, 24(13):1547-1548.

45. Fehrmann RS, et al: Trans-eQTLs reveal that independent genetic variants associated with a complex phenotype converge on intermediate genes, with a major role for the HLA. PLoS Genet 2011, 7(8):e1002197.

46. Fox J: Structural equation modeling with the sem package in R. Structural Equation Modeling-a Multidisciplinary Journal 2006, 13(3):465-486.

47. Smyth GK: Linear models and empirical bayes methods for assessing differential expression in microarray experiments. Stat Appl Genet Mol Biol 2004, 3:Article3.

\section{doi:10.1186/1471-2164-13-636}

Cite this article as: van Eijk et al:: Genetic analysis of DNA methylation and gene expression levels in whole blood of healthy human subjects. BMC Genomics 2012 13:636.

\section{Submit your next manuscript to BioMed Central and take full advantage of:}

- Convenient online submission

- Thorough peer review

- No space constraints or color figure charges

- Immediate publication on acceptance

- Inclusion in PubMed, CAS, Scopus and Google Scholar

- Research which is freely available for redistribution 